\title{
Experiencia en trasplante pulmonar pediátrico en Chile
}

\author{
JOEL MELO T.*, MARÍA T. PARADA C.*, JORGE DREYSE D.*, DAVID LAZO P.*, \\ PATRICIO RODRÍGUEZ D.*, PEDRO F. UNDURRAGA M.*, CRISTIÁN VALVERDE G.*, \\ RODRIGO DÍAZ G.* y CHRISTIAN FAJARDO J.*
}

\section{Experience with pediatric lung transplantation in Chile}

Lung transplantation (TP) is a treatment option in children with terminal lung diseases. $\boldsymbol{O b}$ jective: To evaluate the results and survival of pediatrics lung transplant patients. Methods: Retrospective analysis of clinical records of lung transplantation of patients $\leq 15$ years from Clinica Las Condes, Santiago, Chile. Demographic data, type of transplant, baseline and post transplant lung function, early and late complications and survival rate were analyzed. Results: Nine patients $\leq 15$ years-old were transplanted. The average age at transplant was 12.7 years. The main indication was cystic fibrosis (7 patients). The average BMI was 17.6 and all the patients were with home oxygen therapy. 77\% used extracorporeal intraoperative support. Average baseline FEV $V_{1}$ was $25.2 \%$ with progressive improvement in $\mathrm{FEV}$ of $77 \%$ in the first year. The main early complications were hemorrhage and primary graft dysfunction, while late complications were infections and chronic graft dysfunction. Four patients have died and the estimated 2 years survival was $85 \%$. They achieved school reinsertion and three managed to complete university studies. Conclusion: Lung transplantation is an alternative for children with advanced lung diseases improving their survival and quality of life.

Key words: Pediatrics; lung transplantation; Cystic Fibrosis; Survival rate; primary graft dysfunction; Retrospective studies; Chile.

\section{Resumen}

El trasplante de pulmón (TP) es una opción para pacientes pediátricos con enfermedades pulmonares terminales. Objetivo: Evaluar resultados y sobrevida de pacientes pediátricos trasplantados de pulmón. Métodos: Análisis retrospectivo de registros clínicos de pacientes $T P \leq 15$ años de Clínica Las Condes. Se analizaron datos demográficos, tipo de trasplante, función pulmonar basal y post trasplante, complicaciones precoces y tardias y sobrevida. Resultados: Nueve pacientes $<15$ años de edad se han trasplantado. La edad promedio fue 12,7 años. La principal indicación fue fibrosis quística (7 pacientes). El IMC promedio fue de 17,6 y todos estaban con oxígeno domiciliario. El $77 \%$ utilizó soporte extracorpóreo intraoperatorio. Las principales complicaciones precoces fueron hemorragia y la disfunción primaria de injerto mientras que las tardías fueron principalmente las infecciones y la disfunción crónica de injerto. Cuatro pacientes han fallecido y la sobrevida a dos años fue de $85 \%$. El trasplante les permitió una reinserción escolar y 3 lograron completar estudios universitarios. Conclusión: El trasplante pulmonar es una alternativa para niños con enfermedades pulmonares avanzadas mejorando su sobrevida y calidad de vida.

Palabras clave: Trasplante pulmonar; Pediatría; Fibrosis Quística; Sobrevida; disfunción primaria de injerto; estudios retrospectivos; Chile.

* Centro de Trasplantes Clínica Las Condes. Santiago, Chile. 


\section{Introducción}

Mientras el primer trasplante pulmonar en humanos fue desarrollado por Hardy en el año 1963, el primer trasplante pulmonar (TP) pediátrico lo realizó el grupo de la Universidad de Toronto en el año 1987 en un paciente de 16 años con una fibrosis pulmonar familiar. Actualmente el TP es un tratamiento aceptado para pacientes pediátricos bien seleccionados con enfermedad pulmonar en etapa terminal. La escasez de donantes jóvenes, la relativa renuencia en trasplantar a niños menores de 5 años, una menor prevalencia de patología respiratoria terminal en población pediátrica, y las variaciones en las técnicas quirúrgicas explican en parte que sólo 44 centros a nivel mundial efectúen trasplante pediátrico ${ }^{1,2}$.

La mayoría de los trasplantes pediátricos se realizan en niños entre 11-17 años (72\%), siendo excepcional en menores de 1 año (6 trasplantes el último año). Las indicaciones de TP pediátrico varían con la edad siendo las más frecuentes la fibrosis quística (FQ), la hipertensión arterial pulmonar (HTP) y la bronquiolitis obliterante (BO). En pacientes bien seleccionados, el TP mejora la sobrevida y la calidad de vida con resultados comparables a pacientes adultos. Según la Sociedad Internacional de Trasplante de Corazón y Pulmón (ISHLT por sus siglas en inglés) la sobrevida promedio de pacientes pediátricos es de 5,5 años comparable a la sobrevida de pacientes adultos ${ }^{2}$

En Chile, el único centro que ha efectuado TP en población pediátrica es Clínica Las Condes (CLC). El objetivo de este estudio es describir resultados y sobrevida de pacientes $\leq 15$ años que han sido sometidos a trasplante pulmonar en este centro.

\section{Metodología}

Análisis retrospectivo de registros clínicos de pacientes $\leq 15$ años_trasplantados de pulmón en CLC entre los años 1999 a julio de 2018. Se consignaron datos demográficos, función pulmonar pre-trasplante, enfermedad de base, tipo de soporte extracorpóreo intraoperatorio, días en ventilación mecánica invasiva (VMI), complicaciones médicas y quirúrgicas precoces $(<$ de 3 meses) y tardías, estadía hospitalaria, sobrevida perioperatoria y a 24 meses por método de KaplanMeier, reinserción escolar y causas de muerte en el seguimiento. Este estudio fue analizado y aprobado por el comité de ética de nuestra institución. El diagnóstico de rechazo celular (RCA) y/o humoral $(\mathrm{RH})$ se realizó mediante fibrobroncoscopia (FBC) con biopsia transbronquial, análisis histopatológico, tinción $\mathrm{C} 4 \mathrm{~d}$ en biopsia y presencia de anticuerpos donante específicos, siguiendo las recomendaciones internacionales ${ }^{3}$. Los casos de RCA fueron manejados con bolos de metilprednisolona 3 días y el rechazo humoral con plasmaféresis, inmunoglobulina y Rituximab. El rechazo crónico tipo síndrome de bronquiolitis obliterante (SBO) se definió como una caída progresiva de la función pulmonar desde la mejor espirometría post trasplante habiendo descartado un diagnóstico alternativo ${ }^{4}$. La indicación de trasplante se basó en el consenso de selección de candidatos a trasplante de pulmón de la de la ISHLT $^{5}$. La inmunosupresión incluyó terapia de inducción con Basiliximab y metilprednisolona y la de mantención, prednisona, tacrolimus y micofenolato o azatioprina ${ }^{6}$. La profilaxis antibiótica post trasplante en pacientes en pacientes con FQ se basó en cultivos previos. Hasta el año 2015 en receptores con serología con inmunoglobulina $\mathrm{G}$ positiva para citomegalovirus (IgG CMV+) dejábamos profilaxis con aciclovir por 4 meses y seguimiento con carga viral dejando valganciclovir a pacientes IgG CMV (-) o si presentaban viremia positiva en el seguimiento ( $>2.000$ copias) hasta negativización de ésta, itraconazol por un año y cotrimoxazol forte trisemanal de mantención. Desde el año 2016 usamos voriconazol por 3-4 meses y profilaxis universal con valganciclovir ${ }^{6}$. La FBC se realizó previo al alta y luego ante sospecha de rechazo o lesión de vía aérea. En cada control se realizó hemograma, perfil bioquímico, niveles plasmáticos de inmunosupresores, espirometría, radiografía de tórax y carga viral para CMV. El seguimiento post trasplante fue semanal el primer mes, quincenal hasta el tercer mes, mensual hasta el año y luego trimestralmente.

Para el análisis de medias y porcentajes se utilizó Microsoft Excel 15.0 (2016). Se evaluó sobrevida mediante curva de Kaplan Meier con datos al momento del trasplante $(\mathrm{n}=9)$ y a los 2 años, usando para este cálculo sólo 7 sujetos debido a que 2 trasplantes fueron efectuados a mediados del 2018.

\section{Resultados}

Desde abril de 1999 a julio de 2018 se han realizado $92 \mathrm{TP}$, de los cuales 9 han sido en pacientes $\leq 15$ años. Se excluyeron pacientes pediátricos trasplantados de corazón pulmón $(\mathrm{n}=2)$. El promedio de edad fue de 12,7 (rango 7-15), seis de género femenino. El período de seguimiento 
promedio fue de 50 meses (rango 2-124 meses). La principal indicación de trasplante fue FQ (77\%). Tuvimos un caso de BO posterior a un trasplante de médula ósea y un paciente que debutó con disnea progresiva cuyo estudio concluyó una HTP por hemangiomatosis capilar pulmonar y que por deterioro clínico y falla ventricular derecha se conectó a soporte con oxigenación por membrana extracorpórea (ECMO) como puente para trasplante, realizándose este 75 días después. En el momento del trasplante el IMC promedio fue de $17,6 \mathrm{~kg} / \mathrm{m}^{2}$, todos estaban con terapia con oxígeno domiciliario, 8 con ventilación mecánica no invasiva (VMNI) y uno con ECMO. Desde punto de vista funcional el volumen espiratorio forzado en el primer segundo $\left(\mathrm{VEF}_{1}\right)$ promedio de pacientes con enfermedad obstructiva pretrasplante fue $25,2 \%$ del teórico (rango $18-38 \%$ ) (Tabla 1). En ocho pacientes se realizó técnica bipulmonar; el caso monopulmonar derecho fue efectuado porque la paciente había sido sometida a una neumonectomía izquierda previamente por hemoptisis masiva. Dos pacientes fueron trasplantados con injerto reducido y otros dos de $120 \mathrm{~cm}$ y $140 \mathrm{~cm}$ de talla recibieron trasplantes lobares de donantes adultos

El $88 \%$ de los pacientes requirió soporte extracorpóreo intraoperatorio, 6 casos con ECMO y dos casos con bypass cardiopulmonar (CPB). Seis pacientes continuaron con ECMO en el post operatorio inmediato. El promedio de días en VMI post trasplante fue de 5 días (rango: 2-13) y la estadía hospitalaria promedio fue de 24 días (rango: 14-46) (Tabla 2).

\section{Complicaciones precoces}

La principal complicación quirúrgica fue la hemorragia, requiriendo re- exploración 3 pacientes. También se presentaron un seroma infectado de herida operatoria y un derrame pleural que se resolvió con un drenaje pleural. Dentro de las complicaciones médicas la disfunción primaria de injerto (DPI), una forma de injuria pulmonar post-reperfusión, se observó en 3 pacientes (33\%) (Tabla 3).

\section{Complicaciones tardías}

Hubo 6 episodios de rechazo, 4 RCA y 2 RH, presentándose un 33\% de RCA dentro del primer año, todos manejados en forma satisfactoria.

Se registraron 23 episodios infecciosos, siendo 13 de origen bacteriano principalmente cuadros de bronquitis por pseudomonas y destacando un shock séptico por neumococo; 7 casos por virus comunitarios como influenza, parainfluenza y virus respiratorio sincicial, y 3 infecciones fúngi- cas por aspergillus. Un $91 \%$ de estos cuadros se presentaron después del primer año. En pacientes con seguimiento de más de 2 años un $66 \%$ desarrollo un cuadro clínico de disfunción crónica de injerto (Tabla 3).

\section{Mortalidad y supervivencia}

Durante el seguimiento 4 pacientes han fallecido, una por sangrado intraoperatorio en el trasplante, otro por hemorragia digestiva a los 2 años y 11 meses y otras dos fallecidas, una a los 10 años 4 meses por BOS y otra a los 10 años 11 meses por síndrome linfoproliferativo post trasplante que se manifestó como un linfoma gastrointestinal.

\section{Tabla 1. Características demográficas de pacientes} trasplantados $\leq$ de 15 años: $n=9$

\begin{tabular}{|ll|}
\hline Edad, años (promedio /rango) & $12,7(7-15)$ \\
Género (M/F) & $3 / 6$ \\
$\begin{array}{l}\text { Enfermedad de base } \\
\text { - FQ }\end{array}$ & 7 \\
- HTP & 1 \\
- BO & 1 \\
\% VEF 1 pretrasplante & 25,2 \\
(promedio/rango) & $(18-38)$ \\
IMC (promedio/rango) & $17,6(14,9-21)$ \\
VMNI & 8 \\
ECMO como puente para trasplante & 1 \\
\hline
\end{tabular}

FQ: Fibrosis quística; HTP: Hipertensión pulmonar; $\mathrm{BO}$ : Bronquiolitis obliterante; $\mathrm{VEF}_{1}$ : Volumen espiratorio forzado en el primer segundo; IMC: Índice de masa corporal: VMNI: Ventilación mecánica no invasiva; ECMO: Oxigenación por membrana extracorpórea.

Tabla 2. Datos intra y post operatorios

\begin{tabular}{|c|c|}
\hline $\begin{array}{l}\text { Tipo de trasplante (n) } \\
\text { - Bipulmonar } \\
\text { - Unipulmonar }\end{array}$ & $\begin{array}{l}8 \\
1\end{array}$ \\
\hline $\begin{array}{l}\text { Soporte extracorpóreo (n) } \\
\text { - ECMO } \\
\text { - CPB }\end{array}$ & $\begin{array}{l}6 \\
2\end{array}$ \\
\hline $\begin{array}{l}\text { VMI post trasplante } \\
\text { (días promedio/rango) }\end{array}$ & $2(2-13)$ \\
\hline $\begin{array}{l}\text { Estadía hospitalaria } \\
\text { (días promedio/rango) }\end{array}$ & $24(14-46)$ \\
\hline $\begin{array}{l}\text { Mortalidad perioperatoria } \\
(\mathrm{n} / \%)\end{array}$ & $1(11)$ \\
\hline
\end{tabular}

ECMO: Oxigenación por membrana extracorpórea; CPB: Bypass cardiopulmonar; VMI: Ventilación mecánica invasiva. 
El cálculo de la supervivencia (Kaplan Meier) perioperatoria y a 24 meses fue de $88 \%(8 / 9)$ y $85 \%(6 / 7)$ respectivamente (Figura 1).

\section{Función pulmonar y reinserción escolar}

Todos los pacientes se fueron de alta sin oxígeno. Los 6 pacientes con seguimiento de más de un año retomaron sus estudios y 3 completaron

Tabla 3. Complicaciones médicas y quirúrgicas

\begin{tabular}{|lccc|}
\hline Tiempo & $\begin{array}{c}<\mathbf{3} \\
\text { meses }\end{array}$ & $\begin{array}{c}\mathbf{3 - 1 2} \\
\text { meses }\end{array}$ & $\begin{array}{c}>\mathbf{1 2} \\
\text { meses }\end{array}$ \\
Complicaciones médicas (n) & & & \\
DPI & 3 & & \\
Infecciones & & & \\
$\quad$ Bacterianas & 1 & 1 & 11 \\
$\quad$ Virales no CMV & 0 & & 7 \\
$\quad$ Hongos & 0 & & 3 \\
Insuficiencia renal & 1 & 1 & 1 \\
Rechazo celular & & & \\
Rechazo humoral & & 3 & 1 \\
Complicaciones quirúrgicas (n) & & & \\
$\quad$ Reexploración por & & & \\
Sangrado & 3 & & \\
$\quad$ Derrame pleural & & & \\
$\quad$ Estenosis bronquial & 1 & 1 & \\
Rechazo crónico (n) & & & 4 \\
Mortalidad (n) & 1 & & 3 \\
\hline
\end{tabular}

CMV: Citomegalovirus. estudios universitarios. Desde el punto de vista funcional el $\mathrm{VEF}_{1}$ promedio pre trasplante fue de $25 \%$ del valor teórico (rango: 18-38\%) siendo al año de $77 \%$ (rango: $45-100 \%$ ).

\section{Discusión}

Pocos centros en el mundo efectúan trasplante pediátrico. El único centro en Chile que ha realizado trasplante en niños es Clínica Las Condes, por lo anterior este estudio retrospectivo refleja la realidad nacional y los resultados de pacientes pediátricos trasplantados de pulmón en el país.

Nueve pacientes de edades $\leq 15$ años se han trasplantado desde el año 1999, lo que equivale al 3,4\% de los trasplantes que se han realizado a nivel nacional y a un $9,7 \%$ de los trasplantes efectuados en nuestro centro. El número de centros que realizan TP pediátrico es reducido, con sólo 107 procedimientos realizados el último año en el mundo, una proporción claramente inferior a los 4.500 TP en adultos en la misma fecha, con $72 \%$ de pacientes mayores a 11 años $^{2}$. En nuestra serie la edad promedio al momento del trasplante fue de 12,7 años y en el año 2018 realizamos los primeros TP de menores a 12 años quienes padecían de FQ con edades de 11 y 7 años con buena evolución a la fecha. El principal diagnóstico de base fue FQ $(77 \%)$ similar a la estadística internacional donde sobre los 6 años la principal indicación es $\mathrm{FQ}^{2}$.

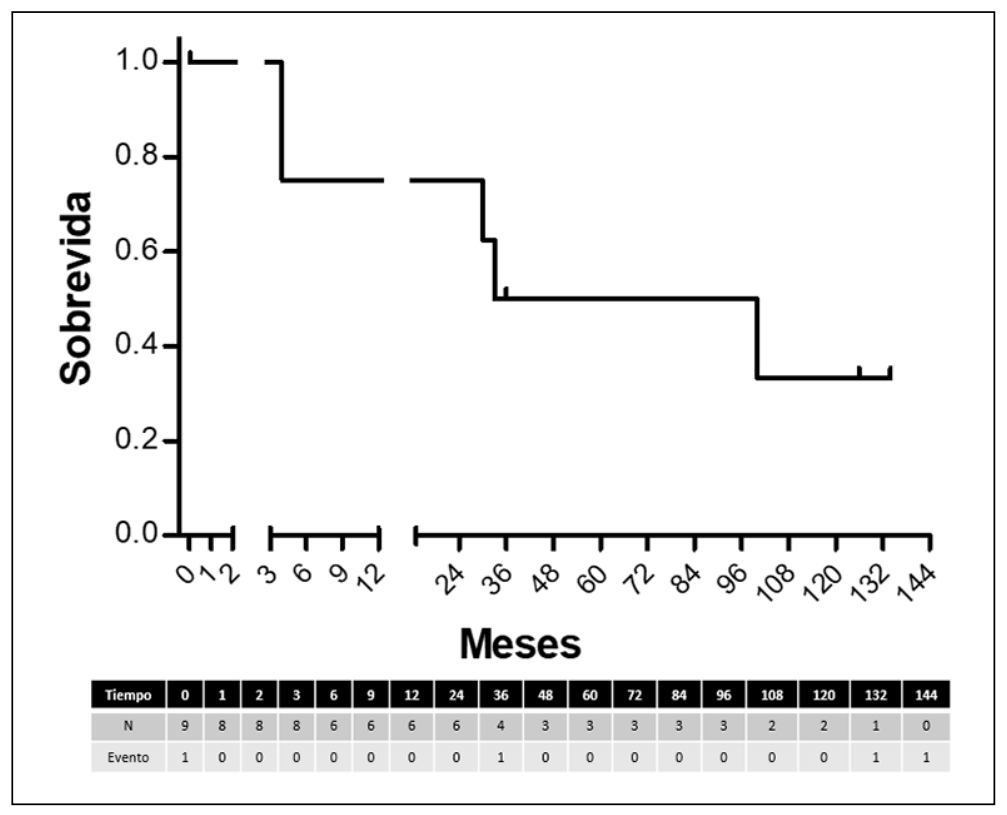

Figura 1. Curva de sobrevida de los pacientes trasplantados de pulmón. 
El principal factor limitante del TP en el mundo es la limitada disponibilidad de órganos y debido a su tamaño, los pacientes pediátricos tienen menor posibilidad de obtener un órgano tamaño compatible con tiempos en lista de espera más prolongados. Dentro de las estrategias para afrontar este problema están las que se mencionan a continuación ${ }^{7}$.

a) TP con donantes vivos relacionados: en el que dos familiares donan no más de un lóbulo pulmonar que luego son implantados en el receptor pediátrico. Este procedimiento se realiza principalmente en Japón donde el tiempo en lista de espera de donantes cadavéricos supera los 800 días, limitando esta cirugía a pacientes severamente enfermos con falla respiratoria progresiva que son incapaces de esperar tiempos largos en lista de espera ${ }^{8}$. En Chile, no se ha realizado este procedimiento.

b) TP de donante cadáver con injerto reducido. En nuestra serie en 2 pacientes se ha realizado este procedimiento, ambos cursaron con DPI moderada a severa y tuvieron que ser reexplorados por sangrado, uno lleva 8 años de seguimiento y el otro falleció a los 2 años 11 meses por hemorragia digestiva.

c) El trasplante lobar bipulmonar con donante cadáver. Los primeros dos casos de este tipo de procedimiento se realizaron el año 2018. El primero una paciente de 7 años de $120 \mathrm{~cm}$ de estatura, que llevaba 16 meses hospitalizada por exacerbación y otra de 11 años de $140 \mathrm{~cm}$ de estatura con deterioro clínico progresivo y un $\mathrm{VEF}_{1}$ del $26 \%$ del valor teórico. En ambas se implantaron ambos lóbulos inferiores de un donante adulto quedando como pulmón derecho e izquierdo respectivamente, no presentaron complicaciones y con estadía hospitalaria de 14 días post $\mathrm{TP}$.

Estas diferentes técnicas quirúrgicas son estrategias seguras y exitosas que benefician al TP pediátrico incrementando la disponibilidad de órganos, disminuyendo la muerte de candidatos en lista de espera, reduciendo las complicaciones postoperatorias relacionadas al tamaño del injerto y con resultados no inferiores al trasplante estándar de donantes con injerto del mismo tamaño9.

A diferencia de los adultos la mayoría de los TP en niños se realizan con circulación extracorpórea, ya que la mayoría de ellos por su tamaño no toleran la ventilación unipulmonar. En nuestra serie el principal sistema de soporte fue el ECMO. El uso de ECMO en trasplante pediátrico ${ }^{9}$ puede plantearse en varias situaciones siempre y cuando el paciente no tenga disfunción multiorgánica.

1) ECMO como puente para trasplante: Tuvimos un paciente de 14 años con HTP por hemangiomatosis capilar pulmonar con falla ventricular derecha rápidamente progresiva que se conectó a ECMO vigil lo que permitió una rehabilitación y mejoría aumentó $8 \mathrm{~kg}$ de peso trasplantándose después de 75 días de soporte.

2) ECMO intraoperatorio: En recientes series la tasa de uso de CPB o ECMO va de un 56 a un $100 \%$, siendo un procedimiento seguro en niños bien seleccionados ${ }^{10}$. Desde el año 2010 en nuestro centro usamos rutinariamente ECMO intraoperatorio en todos los TP pediátricos, logrando disminuir las complicaciones asociadas al CPB y permitiendo una reperfusión más controlada.

3) ECMO post operatorio: se utiliza principalmente en el manejo de la DPI.

En nuestra serie de casos 6 pacientes han continuado con ECMO post trasplante. A excepción de una paciente fallecida por sangrado que requirió ECMO veno-arterial, este tipo de soporte usado los primeros días post trasplante nos ha permitido una reperfusión progresiva del injerto, una estrategia ventilatoria protectora evitando el daño de la ventilación mecánica convencional y manejo de soporte de la DPI. Reconociendo que hay poca literatura al respecto en nuestro centro hemos tenido buenos resultados a corto y mediano plazo.

Las complicaciones infecciosas son causa frecuente de morbilidad y mortalidad en trasplantados pulmonares pediátricos siendo responsables de más del $40 \%$ de las muertes durante el primer año post trasplante 9 . Nosotros registramos 23 episodios en 4 pacientes. Si bien nuestra serie es pequeña, el primer año de seguimiento tuvimos sólo dos pacientes con bronquitis por pseudomonas, número bajo respecto a estadística internacional. Las profilaxis usadas en el post operatorio del trasplante, el seguimiento estricto de los pacientes, las estrategias de vacunación al paciente y familia y la recomendación de no asistir a reuniones sociales ni colegio los primeros 6 meses del trasplante pudieron haber incidido en los resultados. El 91\% de los cuadros infecciosos se presentaron después del primer año. El retorno a sus actividades cotidianas como colegio y la reinserción social predispone a este grupo de pacientes a este tipo de infecciones. La evolución en todos estos casos ha sido satisfactoria y no hemos tenido fallecidos de causa infecciosa. 
La sobrevida perioperatoria y a 24 meses fue de $88 \%(8 / 9)$ y $85 \%(6 / 7)$ respectivamente, resultados a corto y comparables a los reportados en distintos centros que muestran tasas de sobrevida al año de 69 a $90 \%{ }^{11}$. Como centro no trasplantamos a pacientes que estén en VMI convencional que tengan gérmenes de difícil manejo, como Burkholderia, y todos los pacientes en lista de espera se optimiza su condición física con un programa de rehabilitación, además de una intervención nutricional previo al trasplante. La principal limitante de sobrevida a largo plazo es el rechazo crónico ${ }^{9}$, en nuestra serie un $66 \%$ de los pacientes con seguimiento de más de 2 años lo presentaron y sólo un paciente ha fallecido por esta causa.

Uno de los objetivos del trasplante es mejorar la calidad de vida y la sobrevida. El 88\% de los niños no tienen ninguna limitación en sus actividades ${ }^{11}$. En nuestra serie un reflejo de esto fue que los pacientes se fueron de alta sin oxígeno, 6 pacientes con seguimiento de más de un año retomaron sus estudios y 3 completaron estudios universitarios. Además, de 5 pacientes trasplantados desde el 2015, cuatro han sido pacientes del sector público de salud que con un trabajo metódico y multidisciplinario entre los centros hospitalarios ha permitido beneficiar a estos pacientes.

Es un estudio retrospectivo con todas las limitaciones que implica la recolección de datos y el número pequeño de pacientes estudiados, pero al ser un solo centro el que realiza este procedimiento refleja la situación actual del TP pediátrico en Chile.

\section{Conclusión}

La principal indicación de TP pediátrico fue la Fibrosis Quística. Las principales complicaciones precoces fueron el sangrado y la disfunción primaria de injerto, mientras que las infecciones fueron la principal complicación tardía.

\section{Bibliografía}

1.- HUDDLESTON C. Pediatric Lung Transplantation. Curr Treat option in Cardiovasc Med. 2011; 13: 68-78.

2.- GOLDFARB S, HAYES D, LEVVEY B, CHERIKH W, CHAMBERS D, KHUSH KIRAN, et al. International Thoracic Organ Transplant Registry of the International Society for Heart and Lung Transplantation: Twenty-first Pediatric Lung and Heart-Lung Transplantation Report 2018. J Heart Lung Transplant. 2018; 37: 1196-206.

3.- MCMANIGLE W, PAVLISKO E, MARTINU T. Acute cellular and Antibody-Mediated Allograf Rejection. Semin Resp Crit Care Med. 2013; 34: 320-35.

4.- HOVANESSIAN A, WALLACE W, LYNCH J, BELPERIO J. CHRONIC Lung Allograft Dysfunction: Evolving Concepts and Therapies. Semin Resp Crit Care Med. 2018; 39: 155-71.

5.- ORENS JB, ESTENNE M, ARCASOY S, CONTE JV, CORRIS P, EGAN JJ, et al. Pulmonary Scientific Council of the International Society for Heart and Lung Transplantation. International guidelines for the selection of lung transplant candidates: 2006 update-a consensus report from the Pulmonary Scientific Council of the International Society for Heart and Lung Transplantation. J Heart Lung Transplant. 2006; 25: 745-55.

6.- SEPÚLVEDA C, LEIVA V, LINACRE V, SALINAS M, UNDURRAGA P, LÓPEZ J, et al. Trasplante pulmonar en pacientes con Fibrosis Pulmonar. Experiencia del Instituto Nacional del Tórax. Rev Chil Enferm Respir 2019; 35: 15-21.

7.- BENDEN C. Pediatric lung Transplantation. J Thorac Dis 2017; 9: 2675-83.

8.- DATE H. Living-related lung transplantation. J Thorac Dis 2017; 9: 3362-71.

9.- SCHMID F, BENDEN C. Special considerations for the use of lung transplantation in pediatrics. Expert Rev Respir Med 2016; 10: 655-62.

10.- ZUERCHER AJ, INCI I, BENDEN C, FRETZ G, BÉCHIR M, BOEHLER A, et al. Intra-operative extracorporeal membrane oxygenation use in pediatric lung transplantation - The Zurich experience. Pediatr Transplantation 2013; 17: 800-5.

11.- MORENO A, SOLÉ J, ROMAN A. Trasplante pulmonar en niños. Aspectos específicos. Arch Bronconeumol. 2013; 49: 523-8.
Correspondencia a:

Dr. Joel Melo Tanner.

Clínica las Condes.

Lo Fontecilla 441. Las Condes. Santiago, Chile.

Email: joelmelot@hotmail.com 